\title{
FAINT BLUE GALAXIES AND MERGING: THE EVOLUTION OF THE LUMINOSITY FUNCTION
}

\author{
A. CAVALIERE ${ }^{1}$, and N. MENCI ${ }^{2}$ \\ 1 Astrofisica, Dipartimento di Fisica II Università di Roma, \\ via della Ricerca Scientifica 1, I-00133 Roma, Italy. \\ 2 Osservatorio Astronomico di Roma, \\ via dell'Osservatorio, I-00040 Monteporzio (Roma), Italy.
}

\begin{abstract}
$A B S T R A C T$.
We probe to what extent not only the counts of the faint blue galaxies and their redshift distribution but also their $z$-resolved luminosity functions can be explained in terms of binary merging (aggregations).

We present a dynamical theory of such interactions. On this basis we find that "minimal aggregations" taking place within large scale structures and triggering starbursts yield rates and timing such as to explain the observations of the local, flat luminosity function and of its progressive rising and steepening for redshifts out to $z \sim 1$. Correspondingly, we predict faint blue counts still rising up to $m_{B} \sim 28-29$, redshift distributions shifting toward larger $z$ with increasing $m_{B}$; in addition, we predict an upturn of the faint end of the luminosity function more pronounced in clusters than in the field.

We propose that our picture provides the differential dynamics missing in the canonical hierarchical clustering theories. This reconciles with the observations the steep luminosity functions predicted at high $z$ by such theories.
\end{abstract}

Subject headings: cosmology - galaxies: evolution - galaxies: formation. 


\section{INTRODUCTION}

The observations of faint blue galaxies (FBGs) provided statistically significant counts in works by Koo (1986), Tyson (1988), and Cowie et al. (1988). Then an excess became apparent, namely, by a factor $\approx 5$ at magnitudes $B \lesssim 24$ and $\approx 7$ at fainter ones, over the values expected from normal local galaxies filling a critical Friedmann-RobertsonWalker universe. Over the last decade the growing data base concerning FBGs kept challenging the interpretations.

Various contributions to that excess have been discussed, the simplest being based on improved measurements of the local luminosity function (hereafter local LF). These include the correction of the incompleteness by a factor $\lesssim 1 / 2$ in the previous samples of local galaxies, as pointed out by Lilly et al. (1995) and by Ellis et al. (1996), and the consideration of the upturn at $M_{B} \geq-16$ from a flatter Schechter shape pinpointed by Marzke, Huchra, \& Geller (1994). Both these improvements in the local baseline increase, albeit moderately, the expected counts, since the latter are given by the convolution along the line of sight of the LFs weighted with the cosmological volumes.

Meanwhile, it became apparent that toward faint magnitudes blue galaxies make up a fraction of all galaxies which increases both with decreasing luminosity and with increasing redshift $z$ (Koo \& Kron 1992; Colless et al. 1993). Thus, some sort of galaxy evolution must be at work.

Passive luminosity evolution driven by the shift with age of stellar populations would provide the observed blue counts only if helped by the larger volume contributed by widely open cosmologies with $\Omega_{o} \ll 1$ (see Pozzetti, Bruzual, \& Zamorani 1996). A similar result also holds in the red band down to $K=24$ (Cowie et al. 1994; Djorgovski et al. 1996), though the excess is considerably smaller here. Such cosmologies, however, are out of tune with other evidence (summarized, e.g., by Ostriker \& Steinhardt 1995) favoring a flat universe of higher density, i.e., $\Omega_{0} \gtrsim 0.3$ with a cosmological constant $\lambda_{o}=1-\Omega_{o}$.

On the other hand, a constraint was added with the advent of large spectroscopic samples (Cowie, Songaila, \& Hu 1991; Lilly 1993; Colless et al. 1993; Glazebrook et al. 1995) that provided redshift distributions $N(z)$ with a median value as low as $\langle z\rangle \approx 0.5-0.6$ for the identified objects with $B \lesssim 24$. The low value of the redshift where $N(z)$ is peaked rules out strong luminosity evolution in a flat universe; rather, for $z \lesssim 0.5$, the distribution resembles the behavior provided by passive-evolution models with sophisticated color mixes (Koo 1990). The latter, on the other hand, would imply a very substantial high- $z$ tail unless very specific recipes are implemented (see Gronwall \& Koo 1995), which imply mismatches at lower $z$ (see Cowie et al. 1996).

However, recently Lilly et al. (1995) and Ellis et al. (1996) have provided direct evidence of considerable evolution in the resolved luminosity functions over the range $z \approx 0.1-1$. This concerns only the blue objects, while the red ones appear to be already "in place" by $z \approx 1$, and quite similar to typical $L_{*}$ galaxies of the local kind mainly undergoing passive change. The observed evolution appears to be strongly differential, 
being faster for the lower luminosities measured.

Two main interpretations have been proposed for the onset of such a blue, later lineage of galaxies. One (Babul \& Rees 1992; Babul \& Ferguson 1996) holds these to be very small and numerous lumps in which gas collapse and star formation have been delayed by the high level of the UV background prevailing before $z \approx 1$; shortly thereafter, the photoionizing UV flux might decline sharply enough for this constraint to be lifted. Following the initial short starburst, these objects would rapidly fade away.

The other interpretation stems from the widespread local evidence of starbursts associated with galaxy-galaxy interactions (among the vast literature see the recent papers by Zabludoff et al. 1996; Hutchings 1996 and references therein); this maintains that many more, if smaller, blue starbursts are triggered at increasing depths by tidal interactions taking place mainly with or between numerous dwarfs (Lacey \& Silk 1991). Some such interactions are likely to end up in galaxy-galaxy aggregations, i.e., binary merging, also causing considerable number evolution. A phenomenological treatment of these effects has been outlined by Guiderdoni \& Rocca-Volmerange (1991) and by Broadhurst, Ellis, \& Glazebrook (1992).

A dynamical approach to the kinetics of aggregations has been given by Cavaliere \& Menci (1993), motivated to complement the standard hierarchical clustering theory by its intrinsic inadequacy in two specific respects: it predicts too many small objects, and it envisages too little dynamics to erase them; so by itself it would set and preserve a steep luminosity function. A reconciliation between excess counts and a locally flat LF may be sought in terms of star formation processes (see White 1994; Frenk, Baugh, \& Cole 1995), but it still falls short of the full goal even on tuning several parameters. Cavaliere \& Menci (1993) instead, on the basis of the dynamics of aggregations, predicted that $N(M, z)$ and hence $N(L, z)$ should flatten out with $z$ decreasing in the range $z \lesssim 1$.

We are encouraged to pursue our dynamical approach further not only by the recent data concerning the $z$-resolved luminosity functions (which we now compare with) but also by some very recent lines of additional evidence. These include the following: a sizable fraction of FBGs at $z \sim 1$ show dynamical signs of ongoing or recent interactions, or the presence of blue satellites (Koo et al. 1996; Van den Bergh et al. 1996); some FBGs look like spirals with a red bulge (Koo et al. 1996) and peripheral starbursts; widespread post-starburst galaxies mark the action of pervasive galaxy-galaxy interactions (Zabludoff et al. 1996); measurements of clustered redshifts have shown the presence of at least some galaxy concentrations (Le Fèvre et al. 1994; Koo et al. 1996).

Here we will start from first principles and build up a dynamical theory of interactions containing no free parameters once one specifies the environment where the interactions take place. Our main point will be that many such interactions can occur even at moderate redshifts $(z \sim 1)$ within large scale structures that offer the best combination of volume and density contrast. Such interactions, with their mass-dependent cross section $\Sigma(M)$, naturally yield a differential evolution. This affects the dwarf galaxy numbers yielding considerable density evolution, while the associated starbursts affect all blue luminosities yielding a milder luminosity evolution. In terms of observables, 
we find counts still rising at very faint magnitudes, redshift distributions peaked at a value shifting from $z \approx 0.5$ at $B \lesssim 24$ toward $z \approx 1$ for fainter magnitudes, and resolved luminosity distributions which that flatten out from $z \approx 2$ toward a local Schechter-like shape.

The plan of the paper is as follows. In $\S 2$ we present our dynamical framework to compute the mass functions evolving by aggregations. In $\S 3$ we discuss the strong dependence of such evolution on the galaxy environment. We compare three kinds of environments: the homogeneous "field"; the bound, virialized structures; and the largescale structures, which in fact will dominate the observables. In $\S 4$ we describe how from the evolutionary mass distribution we compute the observables: the resolved luminosity distribution $N(L, z)$, the blue counts $N\left(m_{B}\right)$ and the redshift distribution $N(z)$. In $\S 5$ we give our results and predictions for these observables. In the final section we give and discuss our conclusions.

\section{THE MASS FUNCTION FROM BINARY AGGREGATIONS}

\subsection{The dynamics}

A dynamical theory for galaxy evolution directly concerns the mass function $N(M, t)$. The governing equation will be of the general form

$$
\frac{\partial N}{\partial t} \sim \frac{N}{\tau}
$$

where $\tau^{-1}$ is an average evolutionary rate.

In the simplest case when the evolution of a galaxy is not affected by surrounding companions, the rate will not depend on the mass function itself. But this produces self-similar $N(M, t)$ with faint-end slope unchanged in time. A well known instance is constituted by the Press \& Schechter (1974) formula, corresponding to the choice of $\tau$ discussed by Cavaliere, Colafrancesco, \& Scaramella (1991) and by Kitayama \& Suto (1996).

Hence, we consider the case when the evolutionary rate $\tau^{-1}$ does depend on the number of surrounding masses that interact and eventually aggregate. For a total density $n_{g}(t)$ of galaxies, this corresponds to considering $\tau^{-1} \propto n_{g}\langle\Sigma V\rangle$, where $\Sigma$ is the cross section for aggregation and $V$ is the relative velocity of the two aggregating masses; the average runs over the galaxy velocity distribution. The requirement of total mass conservation (see Lucchin 1988) requires a positive and a negative term on the right hand side of equation (2.1), and easily leads to the following Smoluchowski form for the kinetic equation:

$$
\begin{gathered}
\frac{\partial N}{\partial t}=\frac{1}{2} \int_{0}^{m} d m^{\prime} K\left(m^{\prime}, m-m^{\prime}, t\right) N\left(m^{\prime}, t\right) N\left(m-m^{\prime}, t\right) \\
-N(m, t) \int_{0}^{\infty} d m^{\prime} K\left(m, m^{\prime}, t\right) N\left(m^{\prime}, t\right) .
\end{gathered}
$$


Here the kernel $K\left(m, m^{\prime}, t\right)=n_{g}(t)\langle\Sigma V\rangle$ corresponds to the interaction rate for each pair of masses $m, m^{\prime}$, and contains the physics driving the evolution of the distribution $N(m, t)$. The latter is written in its comoving form; the masses $m \equiv M / M_{*}$ are normalized in terms of $M_{*}$, the mass corresponding locally to the standard characteristic luminosity $L_{*}$ (see Peebles 1993).

Each galaxy aggregation comprises two stages: first, the dark matter halos coalesce; second, the baryonic cores may eventually coalesce. The former process requires grazing, weakly hyperbolic encounters for which the cross section is given by (Saslaw 1986, Cavaliere, Colafrancesco, \& Menci 1992, hereafter CCM92)

$\Sigma=\epsilon\left(\frac{V}{v_{m}}\right) \pi\left(r_{m}^{2}+r_{m^{\prime}}^{2}\right)\left[1+\frac{G\left(m+m^{\prime}\right)}{r_{m} V^{2}}\right] \quad$ with $\quad r_{m}=r_{*} m^{1 / 3}, \quad v_{m}=v_{*} m^{1 / 3}$,

where $v_{*}$ and $r_{*}$ are the velocity dispersion and dark-halo radius of the present $M_{*}$ galaxy. We use $M_{*}=510^{11} h^{-1} M_{\odot}{ }^{1} ; r_{*}$ is conservatively taken to be $40 h^{-1} \mathrm{kpc}$ (see Persic, Salucci, \& Stel 1996), and $v_{*}=270 \mathrm{~km} / \mathrm{s}$ (three dimensional). The function $\epsilon\left(V / v_{m}\right)$ describes the decreasing efficiency of the aggregations for increasing relative velocities. Following the results from N-body simulations (see Richstone \& Malumuth 1983) we will take $\epsilon=1$ for $V<3 v_{m}$, and just $\epsilon=0$ for larger values; we shall discuss the effect of such a cutoff in $\S 5$ and $\S 6$. The second term (proportional to $G$ ) in the cross section describes the focusing effect of gravity, an important addition to the purely geometrical cross section proportional to $\pi r_{m}^{2}$ only for large masses and in the range $V<\sqrt{3} v_{m}$ that prevails in long-lived poor groups (see CCM92).

The second stage is constituted by the possible coalescence of the baryonic cores. Sufficient conditions for this to occur have been discussed extensively in terms of dynamical friction of one core against the background provided by the merged halos (see, e.g., Kauffmann, White, \& Guiderdoni 1993). The associated scale $t_{f}$ is at most a factor of 5 longer than the halo crossing time when halo aggregations are effective, namely, for grazing encounters with $V<3 v_{m}$, as discussed above. In all such cases, we find that $t_{f}$ does not exceed the halo aggregation time $\tau$ which drives the evolution of $N(M, t)$ after equation (2.1). Note that such a condition is a sufficient and conservative one, as discussed by Kauffmann et al. (1993), and as confirmed by detailed simulations (including hydrodynamics) of single collisional events and of the ensuing aggregation process (see Barnes \& Hernquist 1991, Barnes 1992).

The solutions of equation (2.2) depend on the setting of the initial conditions, not only as a starting point of the integration procedure, but also - in keeping with the quadratic nature of the right-hand side - for establishing the amplitude of the kernel. We discuss these points in turn.

\subsection{Initial conditions}

The initial conditions are set up at the time $t_{i n}$ corresponding to a redshift $z_{i n}$. Because

1 We parameterize the Hubble constant using $H_{o}=100 \mathrm{~h} \mathrm{~km} / \mathrm{s} / \mathrm{Mpc}$. 
we think of aggregations as complementary to the standard hierarchical clustering (for which see Peebles 1993), we focus on initial mass functions having the Press \& Schechter (1974) form

$$
N(m) d m=\frac{2 a b \delta_{c}}{\sqrt{\pi} M_{*}} \phi(m) d m,
$$

where $\phi(m)=m^{-2+a} \exp \left(-b^{2} \delta_{c}^{2} m^{2 a} / 2\right)$. Here $\delta_{c} \approx 1.68, b^{-1} \sim 1$ fixes the amplitude, and $a=(n+3) / 6$ is related to the spectral index $n \sim-2$ of the power spectrum for the density fluctuations from which galaxies form (see White 1994).

In the same spirit, the typical galactic mass at the initial time $M_{*}\left(t_{i n}\right)$ is identified with the characteristic mass $M_{c}\left(t_{i n}\right)$ provided by the standard hierarchical theory; when normalized to its present value [e.g., $M_{c}\left(t_{o}\right)=0.6 \times 10^{15} h^{-1} M_{\odot}$ for $\Omega=1$ ], this reads (Lupini 1995)

$$
\frac{M_{c}\left(t_{i n}\right)}{M_{c}\left(t_{o}\right)}=\left[\frac{1+3 \frac{\lambda_{o}}{\Omega_{o}} \alpha^{-2}}{\frac{t_{o}^{2}}{t_{i n}^{2}}+3 \frac{\lambda_{o}}{\Omega_{o}} \alpha^{-2}}\right]^{1 / 3 a}
$$

for a density parameter $\Omega_{o}$ and for a cosmological constant satisfying the canonical relation $\lambda_{o}=1-\Omega_{o}$ characteristic of most inflationary scenarios (see Ostriker \& Steinhardt 1995), with $\alpha \equiv \pi H_{o} t_{o} / 2 \Omega_{o}^{1 / 2}$.

In the following, we will consider mainly $b=1$, and $n=$ const $\approx-2.5$ on galactic scales.

The initial mass function (2.4) not only provides the starting condition for equation (2.2), but also affects the numerical value of the kernel at the initial time $t_{i n}$. In fact, we obtain

$$
n_{g}\left(t_{i n}\right)=C \Omega_{o} \rho_{c o} \frac{2 a b \delta_{c}}{\sqrt{2 \pi}} \frac{\left(1+z_{i n}\right)^{3}}{M_{*}\left(t_{i n}\right)} \int \phi(m) d m,
$$

integrated from $M_{*} / 500$ (the smallest mass considered); here $\rho_{c o}$ is the critical cosmological density at the present epoch, and $C$ makes explicit the contrast factor at $t_{i n}$ of the environment where interactions take place.

Note that assuming the Press \& Schechter expression (2.4) and the above values for the parameters is not mandatory; because of the very negative values of the spectral index $n$ at galactic scales, the exponent of the power-law in $\phi(m)$ is always close to -2 , a generic feature of the mass distributions also found in other variants of the hierarchical clustering scenario (see, e.g., White 1994). On the other hand, shortly after $t_{\text {in }}$ the integro-differential nature of equation (2.2) causes the solutions to lose memory of the detailed shape of the initial conditions.

\subsection{Interaction rates}

The numerical value of the interaction kernel $K=n_{g}(t)\langle\Sigma V\rangle$ determines the timescale for the aggregation process. Computing $n_{g}$ as described above, and substituting fiducial 
values for $r_{*}$ and for the initial relative velocity $V_{i n}$, we obtain an expression for $K$ that we conveniently write in the form

$$
\begin{gathered}
K\left(m, m^{\prime}, t\right)=310^{-4} H_{o} t_{o}\left(m^{2 / 3}+m^{2 / 3}\right)\left[1+\frac{v_{*}^{2}}{V^{2}}\left(m^{2 / 3}+m^{2 / 3}\right)\right] \times \\
\left(\frac{r_{*}}{10 \mathrm{kpc}}\right)^{2}\left(\frac{V_{i n}}{100 \mathrm{~km} / \mathrm{s}}\right) n_{g}\left(C, \Omega, t_{i n}\right) f\left(\Omega_{o}, \lambda_{o}, t\right) / t_{o} .
\end{gathered}
$$

The function $f\left(\Omega_{o}, \lambda_{o}, t\right) \equiv n_{g}(t) V(t) / n_{g}\left(t_{i n}\right) V\left(t_{i n}\right)$ describes the change of the kernel after the initial $t_{i n}$ (depending, we recall, on the choice of $\Omega_{o}, \lambda_{o}$ ), and includes the evolution of the relative velocities and that of the density $\rho(t)$ within the environment. The factor $t_{o}^{-1}$ makes explicit the unit of time we use throughout the computation.

The initial density $n_{g}\left(t_{i n}\right)$ is given by equation (2.6). Its value depends on the cosmological scenario under consideration; e.g., for an initial time $t_{\text {in }}$ corresponding to a redshift $z_{i n}=3$ and for a CDM spectrum $(n \approx-2.2$ at galactic scales) with $\Omega=1$ and $C=1$, we obtain $M_{*}\left(t_{i n}\right)=5 \times 10^{11} h^{-1} M_{\odot}$, so that $n_{g}\left(t_{i n}\right) \approx 1.8 h^{3}\left(1+z_{i n}\right)^{3} \approx$ $1.2 \times 10^{2} h^{3} \mathrm{Mpc}^{-3}$ down to $M_{*} / 500$.

The solutions of equation (2.2) are unique (McLeod 1962) once we have specified the initial conditions (through eqs. [2.4] - [2.6]) and the aggregation rate (equation $[2.7])$. We stress that these in turn depend on the following quantities: $C, t_{i n}, V_{i n}$, $f\left(\Omega_{o}, \lambda_{o}, t\right)$; their values describe the environments where aggregations are operating, as will be discussed next.

\section{ENVIRONMENTS}

The kinetic equation (2.2) is naturally written in terms of $t$. In the following we will derive in terms of $z$ the quantities entering this equation and the resulting observables; the conversion from $t$ to $z$ for flat universes may be found, e.g., in Peebles (1993).

\subsection{The homogeneous "field"}

If any such description is realistic - which we doubt in view of the impressive evidence of large scale structures recalled below - the contrast factor should be taken as $C=1$; the density $n_{g} \propto(1+z)^{3}$ decreases after $t_{i n}$ in keeping with the general expansion; the relative velocities, given an initial value, scale as $V \propto(1+z)$. Thus, the aggregation rate changes with $z$ according to

$$
f(z)=\left(\frac{1+z}{1+z_{i n}}\right)^{4}
$$

where $z_{i n}$ is the redshift corresponding to the initial time $t_{i n}$.

The corresponding rapid decrease of $f(t)$ with time makes the aggregation rate rapidly negligible, so that the evolution of the mass function after equation (2.2) will 
become mild at most. Nevertheless, for comparison purposes we compute it using a value for $z_{i n}$ typical of the formation era of galaxies, according to equation (2.5) which in turn depends on $\Omega_{o}, \lambda_{o}$ and on the perturbation spectrum. For a scale-free spectrum with $n=-2.2$ we obtain $z_{i n}=3.5$ when $\Omega=1$ and $\lambda_{o}=0$; and $z_{i n}=4.5$ when $\Omega_{o}=0.2$ and $\lambda_{o}=0.8$. The initial $V_{i n}=V_{o}\left(1+z_{i n}\right)$ is given in terms of the local value $V_{o}$ for the pairwise galaxy velocity dispersion.

The latter was measured first by Davis \& Peebles (1983) (see also Tormen et al. 1993) who found $V_{o} \approx 340 \mathrm{~km} / \mathrm{s}$. Recently Marzke et al. 1995 combined the data from the CfA Redshift Survey and the Southern Redshift Survey and obtained a larger value $\approx 540 \mathrm{~km} / \mathrm{s}$; however, when the Abell clusters with $R \geq 1$ are removed from the sample, the pairwise "velocity dispersion of the remaining galaxies drops to $V_{o}=298 \mathrm{~km} / \mathrm{s}$ ". We adopt the value $300 \mathrm{~km} / \mathrm{s}$. Actually, such a value for $V_{o}$ is conceivably due to the present perturbed gravitational potential; so our scaling back as $1+z$ in this subsection will optimistically maximize the effect, which will be shown to be inadequate anyway.

\subsection{Virialized structures}

Following the standard hierarchical clustering, virialized structures like groups or clusters as environments for galaxy interactions may be characterized simply in terms of the redshift $z$ at virialization. Then, the density contrast is $\sim 180$, the galaxy density scales as $n_{g}(z) / n_{g}(0)=(1+z)^{3}$, and the virial velocity dispersion as

$$
V(z) / V_{o}=(1+z)^{\frac{n-1}{2(n+3)}}
$$

for $\Omega=1$; here the spectral index takes on values in the range $n=-1.5,-1.3$ appropriate for the scale of groups and clusters. Thus, the average aggregation rate would scale as the simple product of these two $z$-dependencies. However, we have to consider the limited survival time of the structure before it is reshuffled into larger and less dense condensations. Such time is evaluated to be $\tau_{p}=3 t / 2$ on average (Cavaliere, Colafrancesco, \& Scaramella 1991; Lacey \& Cole 1993), which corresponds to $\tau_{p}=3 t_{i n}\left(1+z_{i n}\right)^{3 / 2} / 2(1+z)^{3 / 2}$ for $\Omega=1$ and $\lambda_{o}=0$. Including the suppression factor due to limited survival, the effective aggregation rate is $\tau^{-1} \tau_{p} / t_{o}$, and in full reads

$$
n_{g}\langle\Sigma V\rangle \tau_{p} / t_{o}=n_{g}\langle\Sigma V\rangle\left(z_{i n}\right) \frac{3}{2} \frac{t_{i n}}{t_{o}}\left(\frac{1+z}{1+z_{i n}}\right)^{\frac{2 n+1}{n+3}} .
$$

This is equivalent to considering the scaling

$$
f(z)=\left(\frac{1+z}{1+z_{i n}}\right)^{\frac{2 n+1}{n+3}}
$$

and an effective contrast

$$
C=270 t_{i n} / t_{o}
$$


We start our computation at a redshift typical for formation of groups of galaxies, $z_{i n}=2.2$ for an index $n=-1.3$ at the cluster scale. With this value, the initial $V_{i n}$ is evaluated from equation (3.4) on the basis of a (three-dimensional) value $V_{o}=1270$ $\mathrm{km} / \mathrm{s}$ for clusters of richness 1 , to obtain $V_{i n}=480 \mathrm{~km} / \mathrm{s}$. The corresponding effective contrast from equation (3.7) is $C \approx 45$ for $\Omega=1$.

\subsection{Large scale structures}

The large scale structures (LSSs; among the vast literature see De Lapparent, Geller \& Huchra 1992; Sahni \& Coles 1995; Vettolani et al. 1996) constitute an interesting environment for interactions to take place in; over the field they have the advantage of a larger density, and over groups and clusters the advantage of lower velocity dispersions. However, they are quantitatively less understood as yet from both the observational and the theoretical points of view.

In the following, we shall use the semi-analytical approach proposed by Doroshkevich et al. (1995) based on the Zel'dovich approximation and supported by numerical N-body simulations (see again Doroshkevich et al. 1995, and references therein). In such a picture, caustics form first in the matter field at $z_{f} \approx 4$ (for $\Omega=1$ ), then LSSs build up by accretion of matter falling onto them. In this stage aggregations are not effective, because most galaxies have either closely parallel motions or large relative velocities.

Aggregation can be effective in a subsequent stage when the thickness of the pancakes approaches half of their typical separation. This is the epoch $z_{i n}$ when we start our calculations. We evaluate it taking up the results by Doroshkevich et al. (1995), for $\Omega=1$. For $z \lesssim 3$ the mean comoving distance $\mathcal{L}$ between LSSs increases for decreasing $z$ after

$$
\mathcal{L}_{1} \approx r_{c}\left[\frac{8}{3 \pi^{2}} \sqrt{\frac{15}{7}}\left(1+\frac{y^{2}}{2}\right) \exp \left(-\frac{y^{2}}{2}\right)\right]^{-1}
$$

where $y=(1+z) /\left(1+z_{f}\right)$ and $r_{c}$ depends on the power spectrum. The LSS thickness in this stage turns out to be

$$
\ell_{1} \approx 2.2 r_{c} \sqrt{1-\frac{1+z}{1+z_{f}}}
$$

According to the discussion above, we identify $z_{i n}$ with the epoch when $\ell_{1} / \mathcal{L}_{1}$ is $1 / 2$. This yields $z_{i n} \approx 2.3$, independent of $r_{c}$.

The final stage for $z<z_{\text {in }}$ constitutes a comparatively quiet era during which the behavior of the galaxy relative velocities flattens out (Menci \& Valdarnini 1993) to the observed value $V_{i n} \approx 300 \mathrm{~km} / \mathrm{s}$ (see Davis \& Peebles 1983; Tormen et al. 1993).

During this era, the scale of voids evolves as $\mathcal{L}_{2} \approx\left[\sqrt{1+\left[\left(1+z_{t}\right) /(1+z)\right]^{2}}-1\right]^{1 / 2}$ (Doroshkevich et al. 1995), with $z_{t} \approx 0.2$. From such an expression we find the comoving thickness of the structures to scale approximately as $(1+z)^{1 / 2}$ so that the contrast 
behaves like $C(z) \propto(1+z)^{(D-3) / 2}$, where $D$ is the dimensionality of the $\operatorname{LSS}(D=2$ for sheet-like structures, and $D=1$ for filaments). In terms of the function $f(z)$ defined in equation (2.7) this translates to

$$
f(z)=\left[(1+z) /\left(1+z_{i n}\right)\right]^{(3+D) / 2} .
$$

If the local contrast is $\approx 5$, consistent with observations (Geller \& Huchra 1989; Ramella, private communication) and with numerical simulations (Brainerd and Villumnsen 1991; Menci \& Valdarnini 1993), then from the scaling of $C(z)$ we infer an initial contrast $C=5\left(1+z_{i n}\right)^{(D-3) / 2}$, with the value $C \approx 3$ for $D=2$.

In this section we have defined the quantities which enter equation (2.2) for the computation of the evolving mass function in different environments. A summary of the resulting numerical values is given in Table 1. Next we describe how, from the mass function, we compute the observables like the luminosity function, the galaxy number counts and redshift distributions.

\section{FROM N(M,t) TO OBSERVABLES}

Our basic assumption in deriving the observables is that interactions also trigger star formation (see, e.g., Zabludoff et al. 1996, Hutchings 1996, and references therein). Specifically, we conservatively assume that in the range of parameters where aggregations are effective, they also induce and sustain star formation at a rate $s(z)$ equal to the rate of dynamical interactions $\tau^{-1}(z)$ acting on the available gas (see Lacey \& Silk 1991; Broadhurst, Ellis, \& Glazebrook 1992).

The associated luminosity for the dwarf galaxies is given by the relation $L \propto$ $M_{\text {gas }} / \tau$, considering that $\tau$ exceeds the dynamical time of a single galaxy and constitutes the limiting factor. Moreover, the deposition of energy from Supernovae will be initially balanced by cooling, but then for shallow potential wells it will go predominantly into gas expulsion. In this regime, even more directly than in White \& Frenk (1991), the relation $M_{\text {gas }} \propto M v_{m}^{2}$ is obtained. Since $\tau^{-1}$ has the same $M$ and $t$ (or $z$ ) dependencies as given for $K$ by equation (2.7), we obtain

$$
L / L_{*}=\left(M / M_{*}\right)^{\eta} \quad \text { and } \quad L_{*}(z) \propto f\left(z, \lambda_{o}, \Omega_{o}\right),
$$

where $\eta=4 / 3$. Such a value applies when the cross section (2.3) is purely geometrical and is consistent with the observational results by Kormendy (1990). From equation (4.1) the luminosity function $N(L)=N[M(L)] d M / d L$ is obtained.

Since our aim is to isolate the effects of dynamical evolution, we deliberately simplify colors and $k$-corrections to the bones. So we identify the prompt luminosity $L$ with that in the blue band (see Broadhurst et al. 1992), and in the blue spectral region at moderate $z$ we assume a neutral $k$-correction (see Lilly, Cowie, \& Gardner 1991 and Koo \& Kron 1992). We take an absolute characteristic magnitude $M_{B *}=-20$ (see Lilly et al. 1995; Ellis et al. 1996). 
The counts and the redshift distributions are found by convolving the comoving $N(L, z)$ with the cosmological volume $\mathcal{V}$, to obtain

$$
N\left(m_{B}\right) d m_{B}=\int_{0}^{z_{\max }} d z \frac{d \mathcal{V}}{d z}(1+z)^{3} N\left[L\left(m_{b}, z\right)\right] \frac{d L}{d m_{B}} d m_{B}
$$

where the relation between $L$ and $m_{B}$ is given, e.g., by Guiderdoni \& Rocca-Volmerange (1991), and the cosmological volume for a flat universe with $\Omega_{o}+\lambda_{o}=1$ is given, e.g., by Peebles (1993). The range of integration over $z$ extends out to the maximum $z$ from which the magnitude $m_{B}$ can be observed.

\section{RESULTS}

In this section we present our results for the mass distribution, the $z$-dependent luminosity function $N(L, z)$, the counts $N\left(m_{B}\right)$ and the redshift distributions $N(z)$ in different magnitude ranges. These observables are derived from galaxy evolution driven by aggregations acting in the different environments described in $\S 3$.

The plots we present are obtained through the following steps:

i) For a given galaxy environment we compute the aggregation rate given by equation (2.7); the parameters $C, t_{i n}$ (or $\left.z_{i n}\right), V_{i n}$ and the function $f\left(\Omega_{o}, \lambda_{o}, t\right)$ are discussed in $\S 3$, and summarized in Table 1.

ii) We numerically integrate equation (2.2) from the initial time $t_{i n}$ to the present time $t_{o}$. The time step is set at $\Delta t=\left(t-t_{o}\right) / 500$ while the mass step is $M_{*} / 500$.

iii) Following the equations recalled in $\S 4$ we compute $N(L, z), N\left(m_{B}\right)$ and $N(z)$.

The results we obtain are compared with the observations concerning the faint blue counts, the $z$-distributions, and the $z$-resolved LFs.

\subsection{The homogeneous "field"}

For comparison, we put on record the corresponding results, although this is not expected to be an environment conducive to many late binary aggregations due to the rapid decrease of density and velocities.

Figure 1a shows that such is indeed the case for $\Omega=1\left(\lambda_{o}=0\right)$. Because of the fast decrease of $f\left(\Omega_{o}, \lambda_{o}, t\right)$ with time (see Table 1$)$, the interaction rate rapidly becomes small, so that $N(M, t)$ hardly evolves from the initial shape. The corresponding LFs are shown in Figure 1b. Any small evolution that occurs takes place at high redshifts, so that no appreciable change can be observed in the range $z \lesssim 1$. The faint counts in Figure 1c are well fitted, which is not not surprising considering the steep LFs at all redshifts. But the $z$-distributions (Figure $1 \mathrm{~d}$ ) are peaked at $z \approx 1$ even at relatively bright magnitudes.

Thus, aggregations of galaxies uniformly distributed in a FRW universe are uninteresting, and clearly fail to account for the observed evolution in the LFs out to $z \approx 1$. 
One might expect that the plateau (actually the inflection) characteristic of the cosmic scale factor in a Lemaitre universe $(\lambda \neq 0)$ could provide an era of roughly constant density when the binary aggregation processes could proceed for a while. However, in a flat universe such processes are suppressed after the inflection by the accelerated expansion. The net effect is still close to the case $\Omega=1$, as can be seen from Figures $2 \mathrm{a}-2 \mathrm{~d}$ computed for $\Omega_{o}=0.2$ and $\lambda_{o}=0.8$.

\subsection{Virialized structures}

A more favorable environment for galaxy aggregations is constituted by the bound, virialized structures like groups and clusters of galaxies. Here the large density contrast $C$ (though convoluted with the limited survival time discussed in $\S 3.2$ ), and the milder time decrease of the interaction kernel $f\left(\Omega_{o}, \lambda_{o}, t\right)$ yield a sustained evolution of $N(M, t)$ (see Figure 3a) compared to that found for the "field". In Figures $3 \mathrm{~b}-3 \mathrm{~d}$ we show the LFs, the counts, and the $z$-distributions expected if all galaxies resided within virialized structures for a considerable fraction of their lifetime. It is found that the evolution of $N(L, z)$ consists of a rapid flattening in the sub- $L_{*}$ range, with relatively little change in the range from $z \approx 1$ to the present.

It must be noted that in clusters, in spite of the larger value of the contrast $C$, the aggregation rate is suppressed not only by the limited survival time (see $\S 3.2$ ), but also by the increase with decreasing $z$ of the velocity dispersion entering the cutoff $\epsilon\left(V / v_{m}\right)$ in equation (2.3). The latter circumstance has the effect of decreasing the gravitational term in $\Sigma$, and even of shrinking to zero the whole cross section of the smaller galaxies with the lower internal velocities; so at the faint end the mass distribution will hardly be changed from the initial steep shape. The net result will be a LF with an upturn toward the faint end (see Figure 3e). The balance between the effect of the sharp, mass-dependent decrease of the cross section and the general speeding up driven by the large effective contrast is a delicate one, and deserves an aimed study motivated by the LFs recently found (see Driver et al. 1994) to be appreciably steeper in local clusters than in the "field".

\subsection{Large scale structures}

A result in a way intermediate between the two extremes above is provided by the environment constituted by the LSSs, where observations show most galaxies to reside not only locally (Geller \& Huchra 1989), but also at increasingly larger redshifts (see Broadhurst et al. 1990; Schectman et al. 1995; Vettolani et al. 1995; Carlberg et al. 1996). For galaxies inside sheet-like structures (our case $D=2$ ) the parameters entering the aggregation rate are summarized in Table 1 , and yield the evolution of $N(M, t)$ shown in Figure 4a. The corresponding counts and redshift distributions (Figures 4c, 4d) agree with the observations. In particular, they agree with the spectroscopic redshift distribution by Glazebrook at al. (1995) if $L \propto M^{4 / 3}$ holds, and yield an enhanced high$z$ tail for a stronger dependence of $L$ on $M$, e.g., $L \propto M^{1.5}$. The luminosity functions 
(Figure $4 \mathrm{~b}$ ) show a clear flattening from $z \approx 1$ toward a Schechter-like, local shape with $\alpha \approx-1.1$ in agreement with the data.

Note that an upturn at faint luminosities should also be expected here; the mechanism is similar to that discussed at the end of $\S 5.2$, but the effect is considerably milder (and shifted toward fainter luminosities) because the relative velocities inside LSSs are and remain smaller than those in clusters.

Defining a characteristic luminosity by the ratio of the second to the first moment of $N(L, z)$, we find this evolves by about 1 from $z=1$ to $z=0$, as also illustrated in Figure 4a. Actually, the intrinsic luminosity change $L_{*}(z) \propto(1+z)^{(3+D) / 2}$ (see eqs. [4.1] and [3.10]) is nearly balanced by the change following approximately $(1+z)^{-1.5}$, due to characteristic mass after the relation $L \propto M^{4 / 3}$. The net result is a moderate change (about $1 \mathrm{Mag}$ ) of all luminosities, competing with the large change in number for setting the shape of $N(L, z)$. The corresponding $M / L$ ratio evolves (for an $L_{*}$ galaxy) as $M_{*}(z)^{-1 / 3}(1+z)^{-(3+D) / 2}$ which yields $M_{*} / L_{*} \sim(1+z)^{-(2+D) / 2}$.

\section{CONCLUSIONS AND DISCUSSION}

We have derived for FBGs a luminosity function that changes considerably for increasing $z$ as a result of number evolution and a milder luminosity evolution. In particular, in the observed range out to $z \approx 1$ the luminosity function $N(L, z)$ rises and steepens from a local logarithmic slope about -1.1 to a value close to -1.6 at redshift $\approx 0.8$, as observed (see Figure $4 \mathrm{~b}$, and below for a discussion).

This is derived from a dynamical theory of the galaxy mass function based on massconserving binary aggregations. The resulting evolution of $N(M, t)$ is far from being self-similar; indeed, it is strongly differential with mass, causing for smaller galaxies stronger number changes and shift of the mass scale. This constitutes an important complement to the canonical hierarchical clustering, which in fact contains too little dynamics to account for the observed evolution of the luminosity function, as recalled in the Introduction.

The actual strength of the evolution induced by aggregations depends on the environment where these take place. In CCM92 we stressed that in long-lived groups strong, gravitationally focused interactions of large galaxies occur, leading to the formation of cD-like mergers. Here, we find that in the large-scale structures - which comprise most local galaxies, are being observed out to $z \sim 1$, and in a critical universe are expected to loom out at redshifts $z \sim 2$ - small-small and small-large aggregations are favored. Their strength is such as to match - with no special effort at optimization - the existing data for the resolved $N(L, z)$ and for the integrated but deeper observables like counts and magnitude distributions (see Figures $4 a$ to $4 d$ ).

A simple way of summarizing the interaction (and starburst) rates derived in $\S 3.3$ is to note that they scale - differently than in the "maximal merging" model, see Carlberg (1996) - like $\tau^{-1}=\tau_{o}^{-1}\left(M_{*} / M\right)^{1 / 3}(1+z)^{2.5}$. This is because in the rate $\tau^{-1}=n_{g} \Sigma V$ the scaling of the background density $(1+z)^{3}$ is partly offset by the LSS contrast $C(z) \propto(1+z)^{-0.5}$; with mass, on the other hand, the scaling results from $n_{g} \propto M^{-1}$ 
and $\Sigma \propto M^{2 / 3}$. Toomre (1977) has fixed the observed interaction probability $t_{o} / \tau_{o}$ at a few percent for interactions of bright local galaxies; we find $\tau_{o}^{-1}=C n_{g}\left(t_{o}\right) \Sigma V_{o} \approx$ $1 / 500 \mathrm{Gyr}^{-1}$. At $z \approx 1$ our probability $t / \tau$ for galaxies of $5 \times 10^{9} M_{\odot}$ exceeds 30 $\%$. The bright career of this dwarf population activated by interactions is effectively started at $z \approx 2$ when the large scale structures begin to loom out; they reshuffle the conditions set by the canonical hierarchical clustering including the initial correlations of linear perturbations, sustain contrasts larger than unity and produce non-linear relative velocities confined to one or two dimensions. This career terminates, due to the Hubble expansion, with a progressive fading for $z \lesssim 0.5$ and with the number of sub-L* units reduced by $\sim 10^{-1}$.

The LSS features used in our computation - filamentary $(D=1)$ or sheet-like $(D=2$ as considered above) structures produce similar results - come down to three key points: formation era around $z_{i n} \approx 2$, local density contrast $\approx 5$, and slow growth of the contrast from $z_{i n}$ to the present. These features, which appear in the relatively local observations and also in all N-body experiments, are also incorporated in analytic interpretations different from those of Zel'dovich's (see Bond, Kofman, \& Pogosyan 1996). We stress that all such structures are ultimately traced back to long-distance correlations, not considered in the homogeneous renditions of the hierarchical clustering scenario, but actually intrinsic, and effective in bringing to interact galaxies that otherwise would not have met.

The detailed value of the initial contrast $C$ is the only "free" parameter at present. In fact, its magnitude is constrained by its very definition to be of the order of a few, as we have used. As to finer adjustments, its local distribution is currently being measured (Ramella, private communication); the analytic theory of its evolution is being refined, beyond the approximation used in $§ 3.3$ (see Sahni \& Coles 1995); observations are reaching beyond $z \approx 1$ (see Willinger et al. 1996).

Our results are conserved when the shape of the initial mass function is changed from the detailed Press \& Schechter form but is still steep as predicated by all hierarchical clustering scenarios (cf. White 1994).

The main trends are also robust with respect to variations of the $M / L$ ratio from the value $M / L \propto M^{-1 / 3}$ used in $\S \S 4$ and 5 , to include $M / L \propto$ const. and $M / L \propto M^{-2 / 3}$ as shown in Figure 5. The different $M / L$ ratios tilt, but moderately, the shape of the distant LFs; correspondingly, the tail of $N(z)$ for $z>1$ rises to the values indicated by the data by Cowie et al. (1996) if the dependence of $M / L$ goes toward $M^{-2 / 3}$.

We also computed the counts in the $K$-band. These, as expected, increase considerably less than in the blue due to the long evolutionary times of the red stars and to the stronger $k$-correction.

One recurrent objection to the aggregation scenario concerns the correlation function, as discussed by Efstathiou (1995). This is a delicate issue, as is shown in Figure 6. At the statistical level of the correlation function, aggregations with cross section less than linear in mass (like $\Sigma \propto M^{2 / 3}$ for nearly geometrical cross sections that apply here, see $\S 2$ ) do not cause the slope of the initial correlation function to change. A steepening instead takes place for the stronger, gravitationally focussed interactions 
with non-linear cross section $\Sigma \propto M^{4 / 3}$. The whole issue may peter out, however, with the growing evidence for clustered redshifts at $z \approx 0.8-1$ as observed and discussed by Le Fèvre et al. (1994) and by Koo et al. (1996).

The other recurrent objection to aggregations is that the spiral disks may be damaged by merging, as discussed by Ostriker (1990). In our framework, however, at the level of $M_{*}$ the mass growth is due to minor episodes of merging with the numerous but much smaller (and often more diffuse) galaxies. On the other hand, the overall change of the characteristic mass seen in Figure 4a is mainly due to the changing shape of the mass function, i.e., to its flattening corresponding to the disappearance of small galaxies. This results in a mild statistical change in time of the second moment of the distribution, which does not imply a corresponding change in the masses of individual galaxies. For similar reasons, the population of ellipticals does not evolve appreciably by aggregations in the redshift range considered here.

To conclude, excess counts at $B \lesssim 24$ may result from the combination of several effects: local shape and normalization of the luminosity function, long line-of-sight associated with $\Omega_{o}<1$, passive evolution, and interactions as stressed here. The shape of $N(z)$ is more telling, and at relatively bright magnitudes $m_{B} \lesssim 24$ requires some evolution, yet it rules out the pure luminosity kind in a flat universe. The resolved $N(L, z)$ with its evolving shape restricts the issue to the following alternatives: luminositydependent luminosity evolution or a combination of (differential) density plus luminosity evolution, as naturally provided by aggregations. The steepening associated with the latter drives a specific differential shift of $N(z)$, characterized by the peak marching on toward higher $z$ at fainter magnitudes (see Figure $4 d$ ); it also sustains the rise of the counts at very faint magnitudes (see Figure 4c) even beyond $b_{j}=27$ measured by Metcalfe et al. (1995), probing in an integrated fashion the driver's action at redshifts not yet reached by spectroscopy.

The above predictions stem from simple dynamics in which interactions produce aggregations and starbursts together. On the other hand, Cavaliere \& Menci (1993) and Moore et al. (1996) point out that in clusters higher densities but larger relative velocities cause more frequent but weaker encounters ("harassment") resulting mainly in pure starbursting and in blueing of the Butcher-Oemler (1984) type. In fact, such flybys and the true aggregations constitute two facets of a single process, differing as for the detailed form of the cross section (see equation 2.3) and for the ratio $s(z) / \tau^{-1}(z)$ of the star formation to the interaction rates.

These two facets stem from the same dynamics, and concur to stress the importance of interactions as drivers of galaxy evolution. Actually, the intermediate regime should also be observable in clusters, in the form of a local LF frozen with a steeper faint end or a more pronounced upturn, as shown by Figure 3e.

We have benefited from many informative discussions with G. De Zotti, M. D'Onofrio, E. Giallongo, M. Ramella, R. Windhorst and G. Zamorani. We thank the referee for his helpful comments which improved our presentation. This work was supported by partial grants from MURST and ASI. 


\section{REFERENCES}

Babul, A., \& Rees, M.J. 1992, MNRAS, 255, 346

Babul, A., \& Ferguson, H.C. 1996, ApJ, 458, 100

Barnes, J.E., \& Hernquist, L.E. 1991, ApJ, 370, L65

Barnes, J.E. 1992, ApJ, 393, 484

Bond, J.R., Kofman, L., \& Pogosyan, D. 1996, Nature, 380, 603

Brainerd, T.G., \& Villumsen, J.V. 1992, ApJ, 394, 409

Broadhurst, T.J., Ellis, R.S., Koo, D.C., \& Szalay, A.S. 1990, Nature, 311, 726

Broadhurst, T.J., Ellis, R.S., \& Glazebrook, K. 1992, Nature, 355, 55

Butcher, H., \& Oemler, A. 1984, ApJ, 285, L45

Carlberg, R.G. 1996, in "Galaxies in the Young Universe", MPI Conference, ed. H. Hippelein (Springer), preprint

Carlberg, R.G., Cowie, L.L., Songaila, A., \& Hu, E.M. 1996, preprint astro-ph/9605024

Cavaliere, A., Colafrancesco, S., \& Scaramella, R. 1991, ApJ, 380, 15

Cavaliere, A., Colafrancesco, S., \& Menci, N. 1992, ApJ, 392, 41 (CCM92)

Cavaliere, A., \& Menci, N. 1993, ApJ, 407, L9

Colless, M.M., Ellis, R.S., Broadhurst, T., Taylor, K., \& Hook, R.N. 1993, MNRAS, 261,19

Cowie, L.L., Lilly, S.J., Gardner, J.P., \& McLean, I.S. 1988, ApJ, 332, L29

Cowie, L.L., Gardner, J.P., Hu, E.M., Songaila, A., Hodapp, K.W., \& Wainscoat, R.J. 1994, ApJ, 434, 114

Cowie, L.L., Songaila, A., \& Hu, E.M. 1991, Nature, 354, 460

Cowie, L.L., Songaila, A., Hu, E.M., \& Cohen, J.G. 1996, AJ in press astro-ph/9606079

Davis, M., \& Peebles, P.J.E. 1983, ApJ, 267, 465

de Lapparent, V., Geller, M.J., \& Huchra, J.P. 1992, ApJ, 369, 273

Djorgowski, S. et al. 1996, ApJ, 438, L13

Doroshkevich, A.G., Fong, R., Gottlöber, S., Mücket, J.P., \& Müller, F. 1995, preprint

Driver, S.P., Phillips, S., Davies, J.I., Morgan, I., \& Disney, M.J. 1994, MNRAS, 266, 155

Efstathiou, G.P.E. 1995, MNRAS, 272, L25

Ellis, R.S., Colless, M., Broadhurst, T., Heyl, J., \& Glazebrook, K. 1996, MNRAS, 280, 235

Frenk, C.S., Baugh, C.M., \& Cole, S. 1995, in Proc. of 'Mapping, Measuring and Modelling the Universe', Valencia, to appear

Geller, M.J., \& Huchra, J.P. 1989, Science, 246, 897

Glazebrook, K., Ellis, R.S., Colless, M.M, Broadhurst, T.J., Allington-Smith, J.R., Tamvir, N.R., \& Taylor, K. 1995, MNRAS, 273, 157

Gronwall, C. \& Koo, D.C., 1995, ApJ, 440, L1

Guiderdoni, B., \& Rocca-Volmerange, B. 1991, A\& A, 252, 435

Kauffman, G., White, S.D.M, \& Guiderdoni, B., 1993, MNRAS, 264, 201

Kitayama, T., \& Suto, Y. 1996, MNRAS, 280, 638

Koo, D.C. 1986, ApJ, 311, 651 
Koo, D.C. 1990, in ASP Conf. Ser. 10, 'Evolution of the Universe of Galaxies - The Edwin Hubble Centennial Symposium', Aed. R.G Kron (San Francisco: ASP), 268 Koo, D.C., \& Kron, R.G. 1992, ARAA, 30, 613

Koo, D.C. et al. 1996, ApJ, 469, 535

Kormendy, J. 1990, in ASP Conf. Ser. 10, 'Evolution of the Universe of Galaxies', ed. R.G. Kron (San Francisco: ASP), 33

Jones, L.R., Fong, R., Shanks, T., Ellis, R.S., \& Peterson, B. 1991, MNRAS, 249, 481

Hutchings, J.B. 1996, ApJ, 111, 712

Lacey, C., \& Cole, S. 1993, MNRAS, 262, 627

Lacey, C, \& Silk, J. 1991, ApJ, 381, 14

Le Fèvre, O., Crampton, D., Hammer, F., Lilly, S.J., \& Tresse, L. 1994, ApJ, 423, L89

Lilly, S.J., Cowie, L.L., \& Gardner, J. 1991, ApJ, 369, 79

Lilly, S.J. 1993, ApJ, 411, 501

Lilly, S.J., Tresse, L., Hammer, F., Crampton, D., \& Le Fèvre, O. 1995, ApJ, 455, 108

Lucchin, F. 1988, in Lecture Notes in Physics, 332, 'Morphological Cosmology', ed. P. Flin (Berlin: Spriger), 284

Lupini, F. 1996, II Rome University Thesis

Maddox, S.J., Sutherland, W.J., Efstathiou, G., Loveday, J., \& Peterson, B.A. 1990, MNRAS, 247, 1p

Marzke, R.O., Huchra, J.P., \& Geller, M.J. 1994, ApJ, 428, 43

Marzke, R.O., Geller, M.J., da Costa, L.N., \& Huchra, J.P. 1995, AJ, 110, 477

McLeod, J.V. 1962, Quart. J. Math. Oxford, 13, 119

Menci, N., Colafrancesco, S., \& Biferale, L. 1993, Journ. de Physique, 3, 1105

Menci, N., \& Valdarnini, R. 1993, ApJ, 436, 559

Metcalfe, N., Shanks, T., Fong, R., \& Roche, N. 1995, MNRAS, 273, 257

Moore, B. Katz, N., Lake, G., Dressler A., \& Oemler, A. 1996, Nature, 379, 613

Ostriker, J.P. 1990, in ASP Conf. Ser. 10, "The Evolution of the Universe of Galaxies", ed. R. Kron (San Francisco: ASP), 25

Ostriker, J.P., \& Steinhardt, P. 1995, Nature, 377, 600

Peebles, P.J.E. 1993, Principles of Physical Cosmology (Princeton: Princeton Univ. Press)

Persic, M., Salucci, P., \& Stel, F. 1996, MNRAS, 281, 27

Pozzetti, L., Bruzual, G., \& Zamorani, G. 1996, MNRAS, 281, 953

Press, W.H., \& Schechter, P. 1974, ApJ, 187, 425 (P\&S)

Richstone, D.O., \& Malumuth, E.M. 1983, ApJ, 268, 30

Sahni, V., \& Coles, P. 1995, Phys. Rep., 262, 1

Saslaw, W.C. 1985, 'Gravitational Physics of Stellar and Galactic Systems' (Cambridge: Cambridge Univ. Press), 231

Shectman, S.A., Landy, S.D., Oemler, A., Tucker, D.L., Kirshner, R.P., Lin, H., \& Schechter, P.L. 1995, in "Wide Field Spectroscopy and the Distant Universe", in Proc. of 35th Herstmonceaux Conf., eds. S.J. Maddox and A. Aragon-Salamanca (Singapore: World Scientific), 99 
Toomre, A. 1977, in 'Evolution of Galaxies and Stellar Populations', ed. B.M. Tinsley \& R.B. Larson (New Haven: Yale Univ. Observatory), 401

Tormen, G., Moscardini, L., Lucchin, F. \& Matarrese, S. 1993, ApJ, 411, 16 Tyson, A.J. 1988, AJ, 96, 1

van den Bergh, S., Abraham, R.G., Ellis, R.S., Tanvir, N.R., Santiago, B.X., \& Glazebrook, K. 1996, AJ, 112, 359

Vettolani, G. et al. 1995, in "Wide Field Spectroscopy and the Distant Universe", in Proc. of 35th Herstmonceaux Conf., eds. S.J. Maddox and A. Aragon-Salamanca (Singapore: World Scientific), 115

White, S.D.M., \& Frenk, C.S., 1991, ApJ, 379, 52

White, S.D.M. 1994, preprint MPA 831

Willinger, G.M., Hazard, C., Baldwin, J.A., \& McMahon, R.G. 1996, ApJS, in press Zabludoff, A.I., Zaritsky, D., Lin, H., Tucker, D., Hashimoto, Y., Schectman, S.A., Oemler, A., \& Kirshner, R.P. 1996, ApJ, 466, 104 


\section{FIGURE CAPTIONS}

Figure 1: Results for galaxies with $M / L \propto M^{-1 / 3}$ interacting in the homogeneous "field" with $\Omega=1$ and $\lambda=0$.

Panel a): The solid lines show the comoving mass function at 10 equally spaced time intervals, from the initial time $t_{i n}$ (dashed line) to the present.

Panel $\mathrm{b}$ ): The corresponding luminosity functions in the $\mathrm{B}$ band for three redshift bins: $z=0-0.2,0.2-0.5$ and $z>0.5$; the data are taken from Ellis et al. 1996 .

Panel c): The computed galaxy counts (solid line), compared with data from various authors: Maddox et al. (1990) solid circles; Jones et al. (1991), open triangles; Tyson (1988), open circles; Lilly, Cowie \& Gardner (1991), open stars; Metcalfe et al. (1995), solid triangles.

Panel d): The computed redshift distribution in the magnitude range $22.5<m_{B}<$ 24 is compared with the data from Glazebrook et al. (1995).

Figure 2: Same as Figure 1 but for $\Omega_{o}=0.2$ and $\lambda_{o}=0.8$.

Figure 3: Same as Figure 1 but for galaxies within virialized structures.

Figure 3e: The effect of varying the position of the cutoff for the efficiency $\epsilon\left(V / v_{m}\right)$ in the cross section (2.3). In this figure $\epsilon=0$ is taken for $V>2.5 v_{m}$ rather than $V>3 v_{m}$ as adopted throughout the paper.

Figure 4: Same as Figure 1 but for galaxies within large scale structures with $D=2$. In panel $\mathrm{b}$ ) we show also our prediction for the luminosity function at $z=1$ (dotdashed line). In panel c) we show also our predictions for $24<m_{B}<26$.

Figure 5: The evolution of the luminosity function of interacting galaxies within large scale structures, computed for $M / L=$ constant (left panel) and for $M / L \propto M^{-2 / 3}$ (right panel).

Figure 6: The time evolution of the slope $\gamma$ of the two-point correlation function for aggregating galaxies, as computed from a typical Montecarlo simulation (see Menci, Colafrancesco, \& Biferale 1993 for details); $\gamma_{i n}$ is the initial value. The dotted line shows the result for a cross section scaling as $\Sigma \sim M^{4 / 3}$, and the solid line that for $\Sigma \sim M^{2 / 3}$. 
Fig. 1
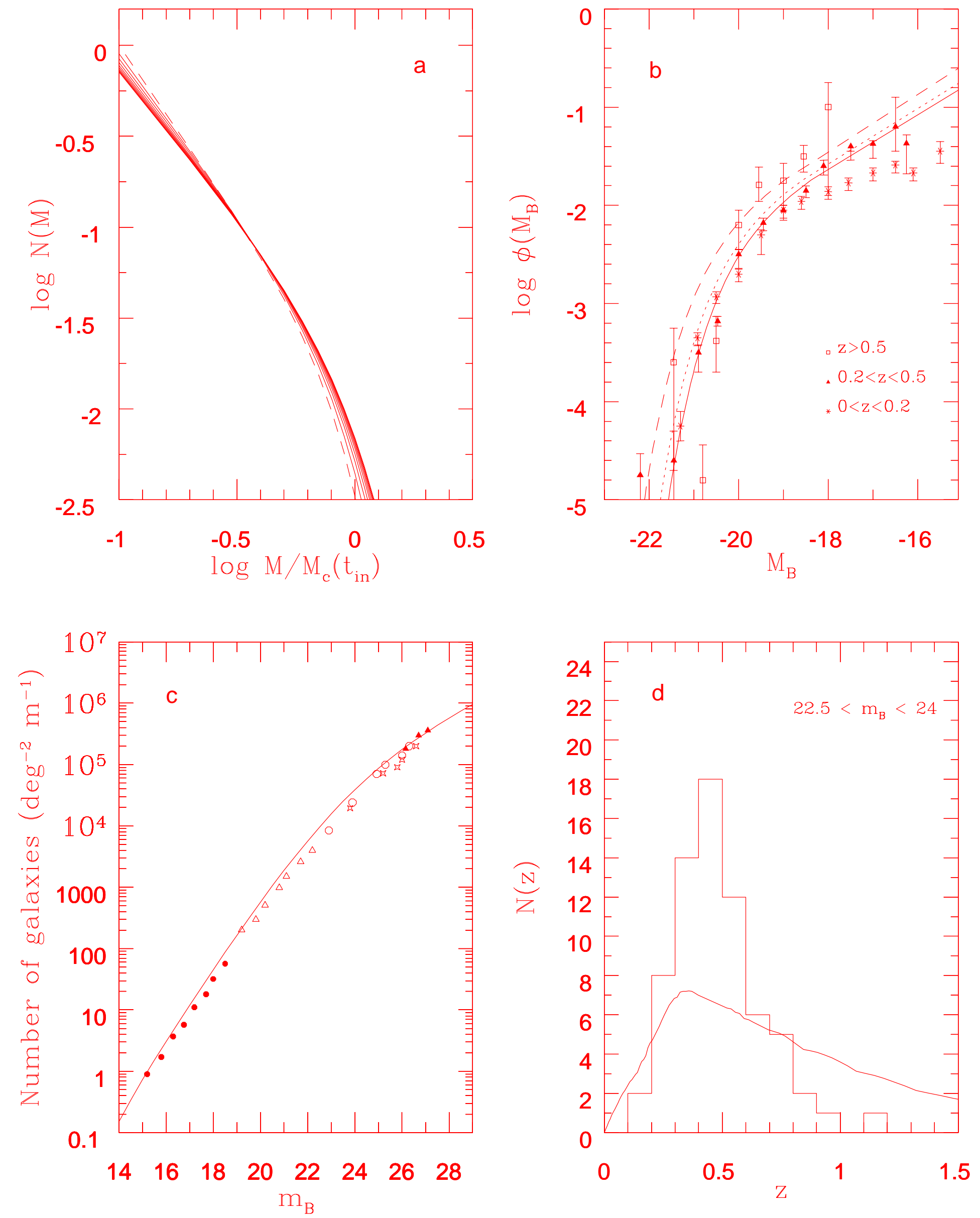
Fig. 2
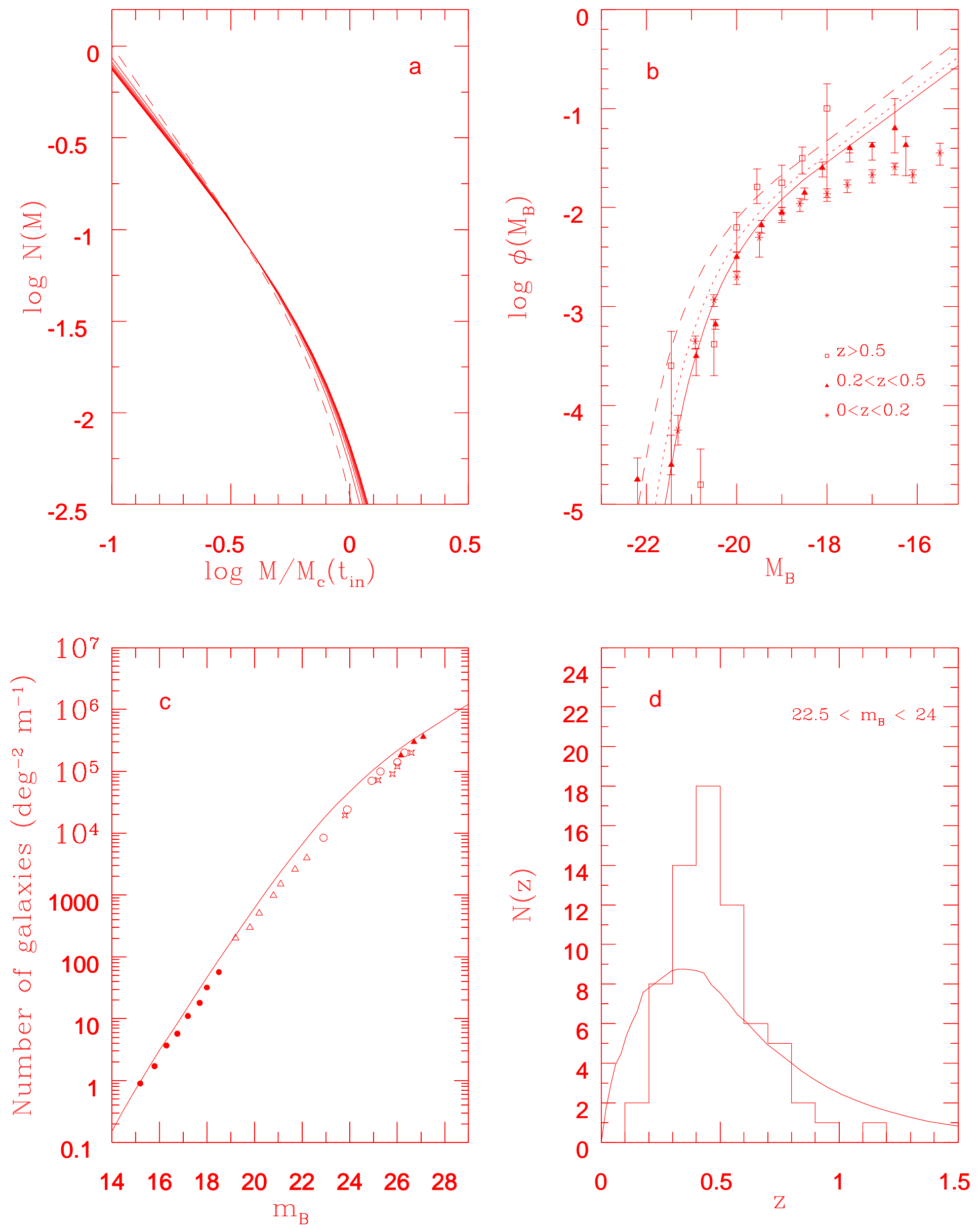
Fig. 3
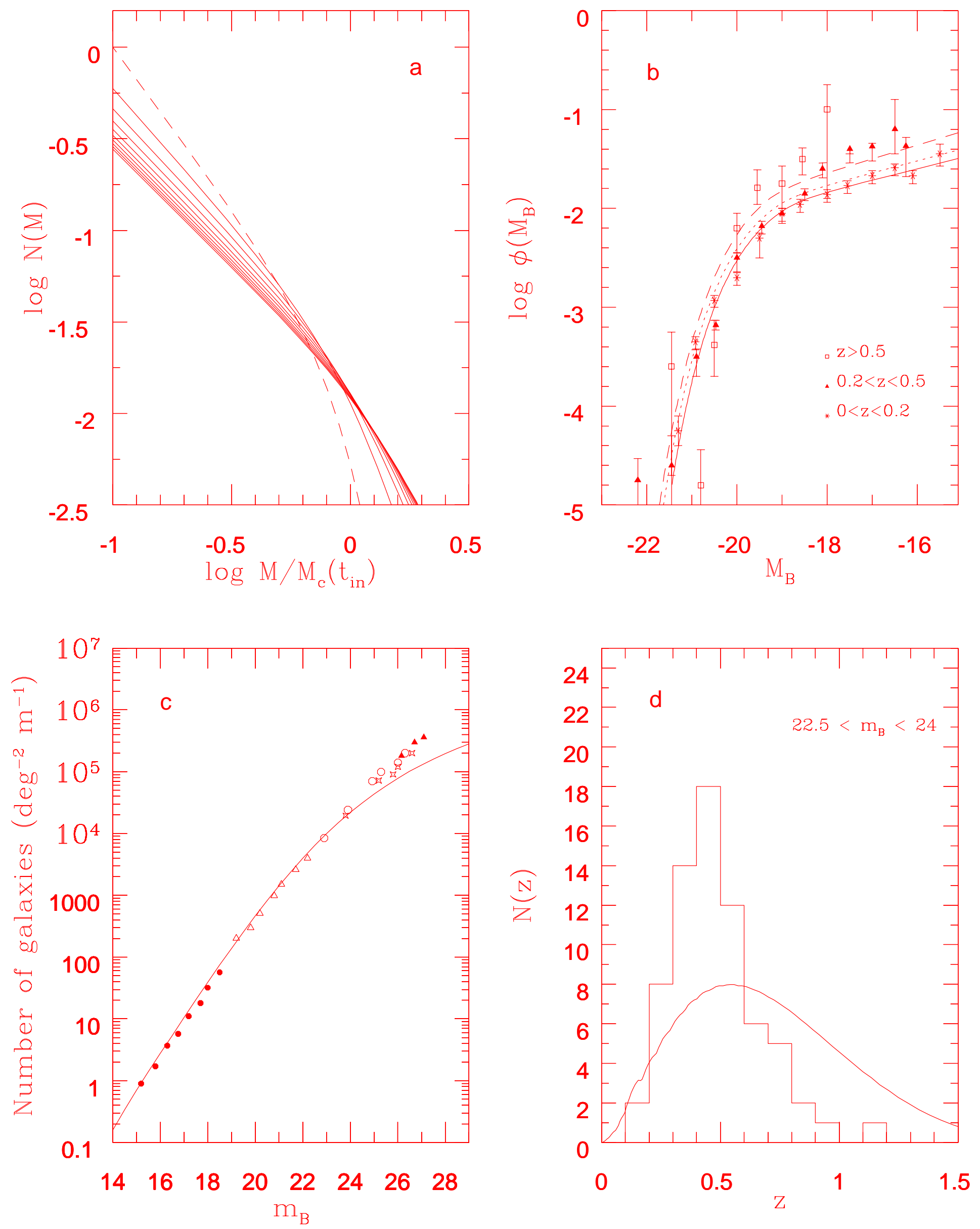
Fig. $3 e$

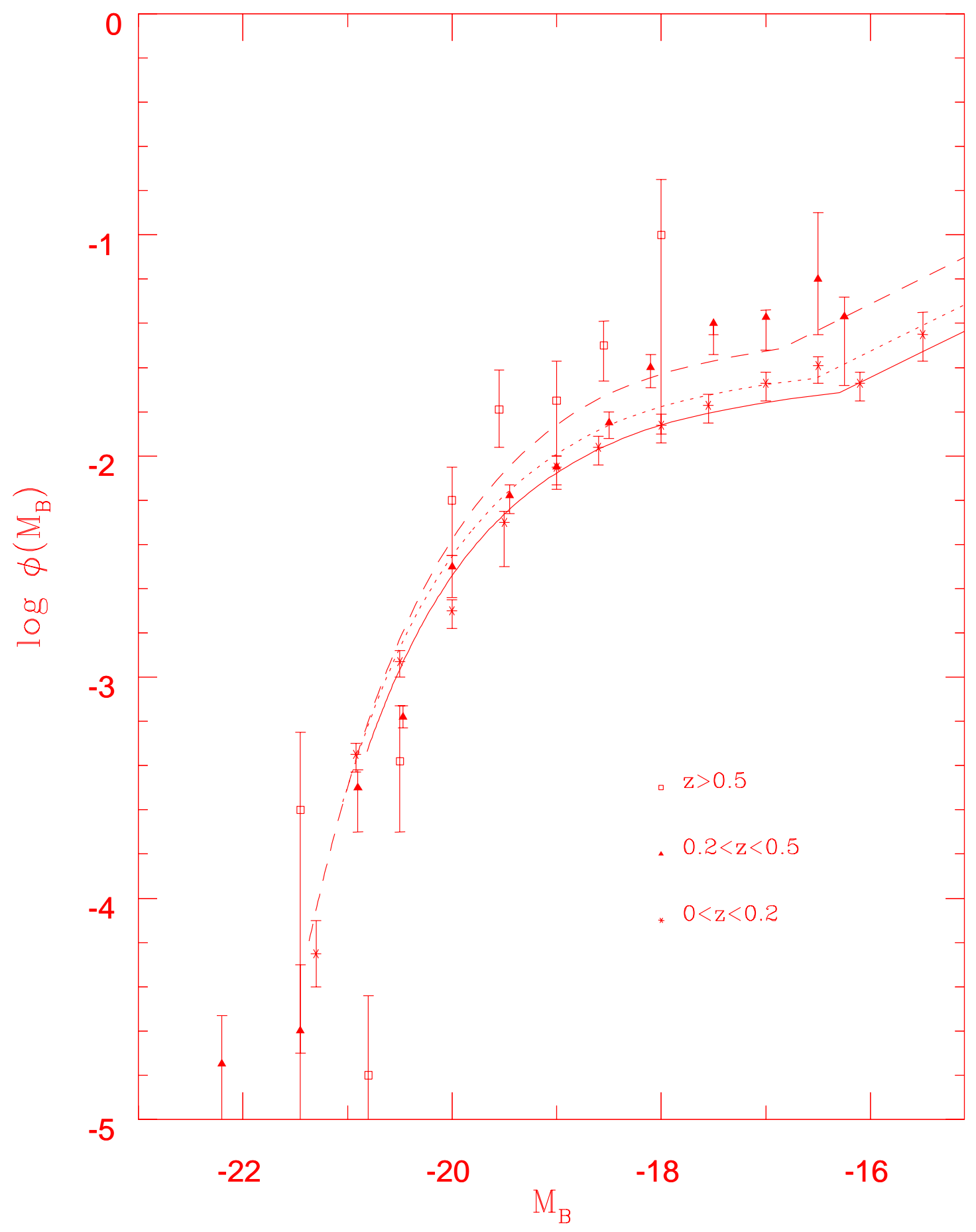


Fig. 4
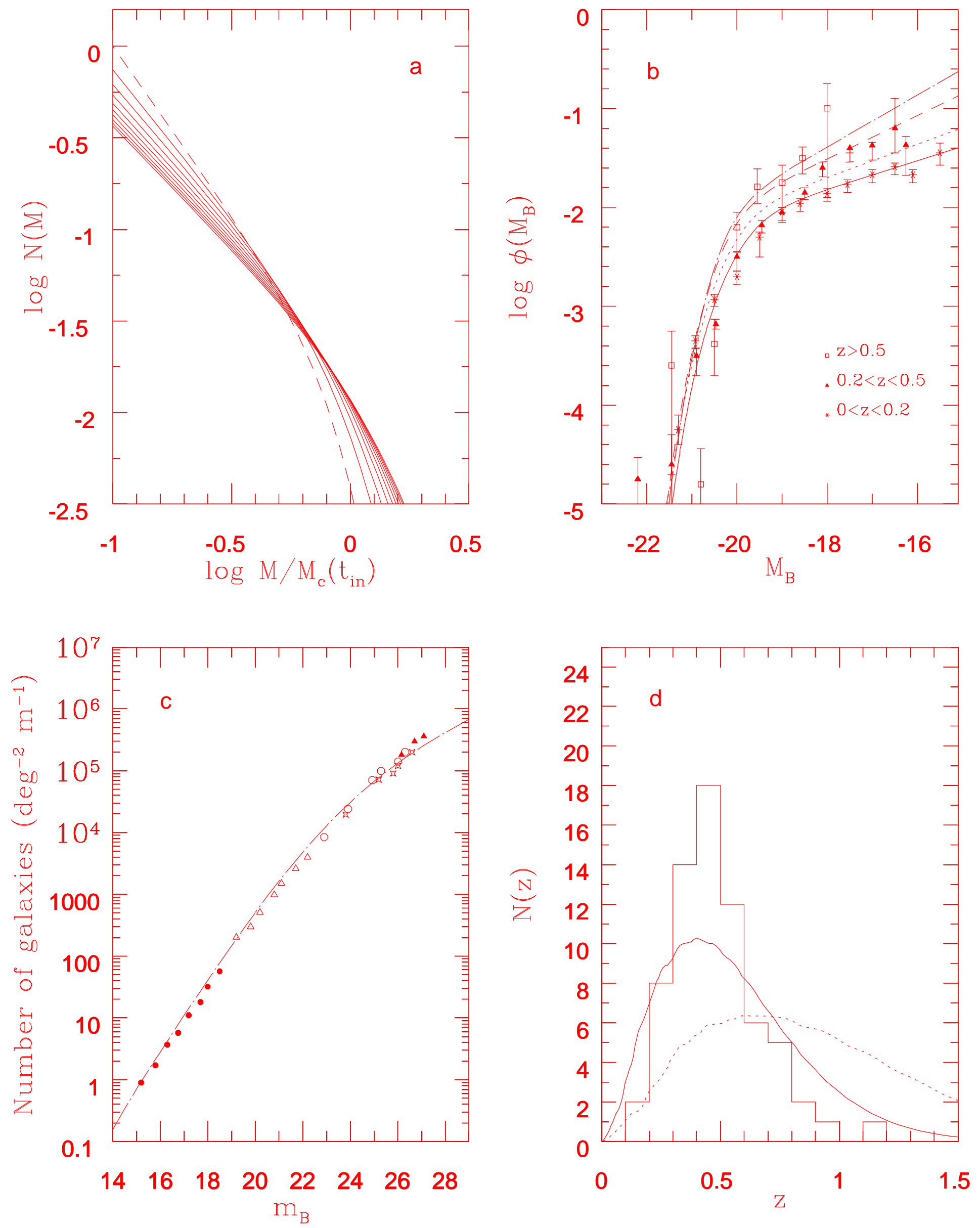
Fig. 5
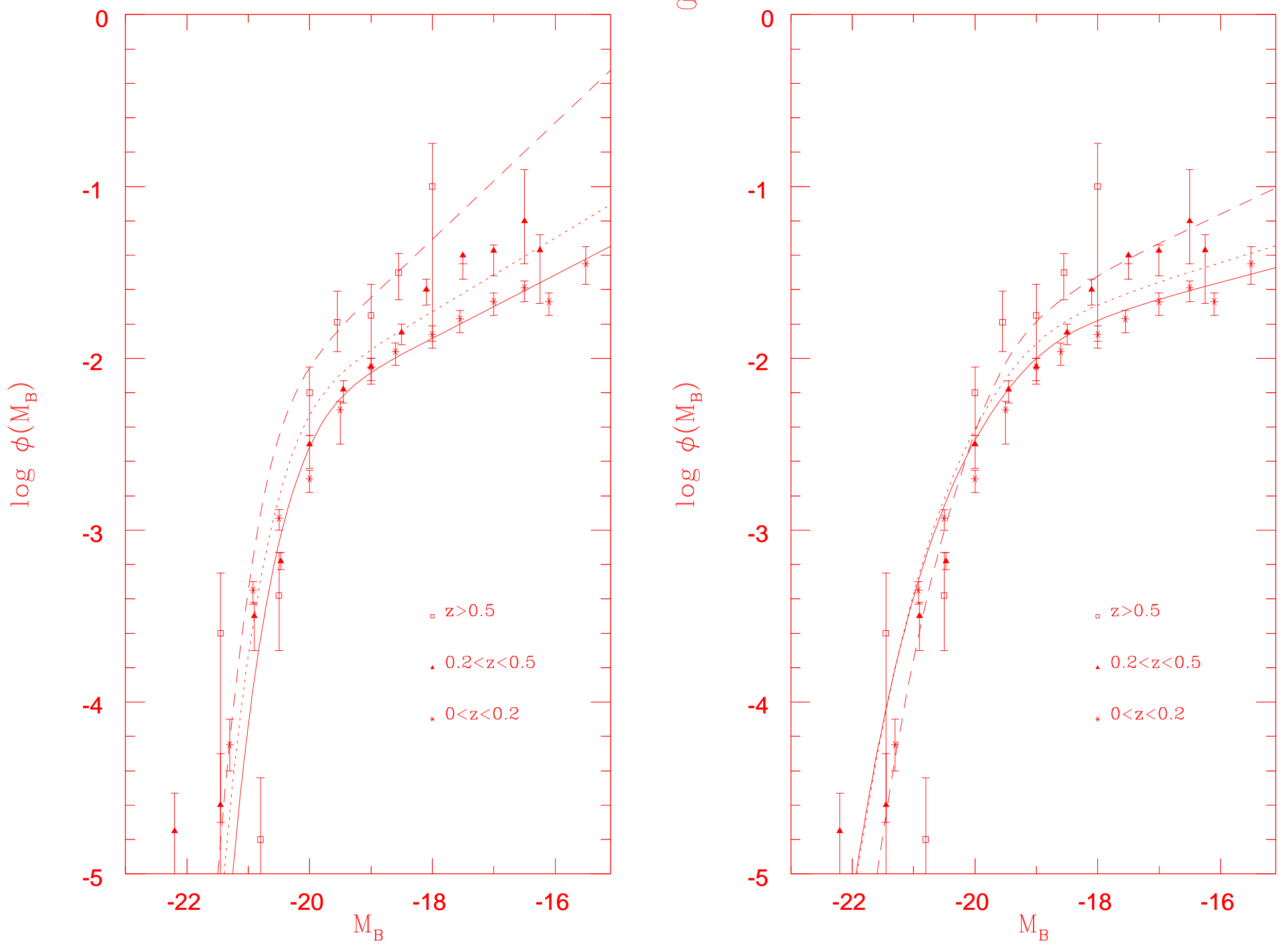


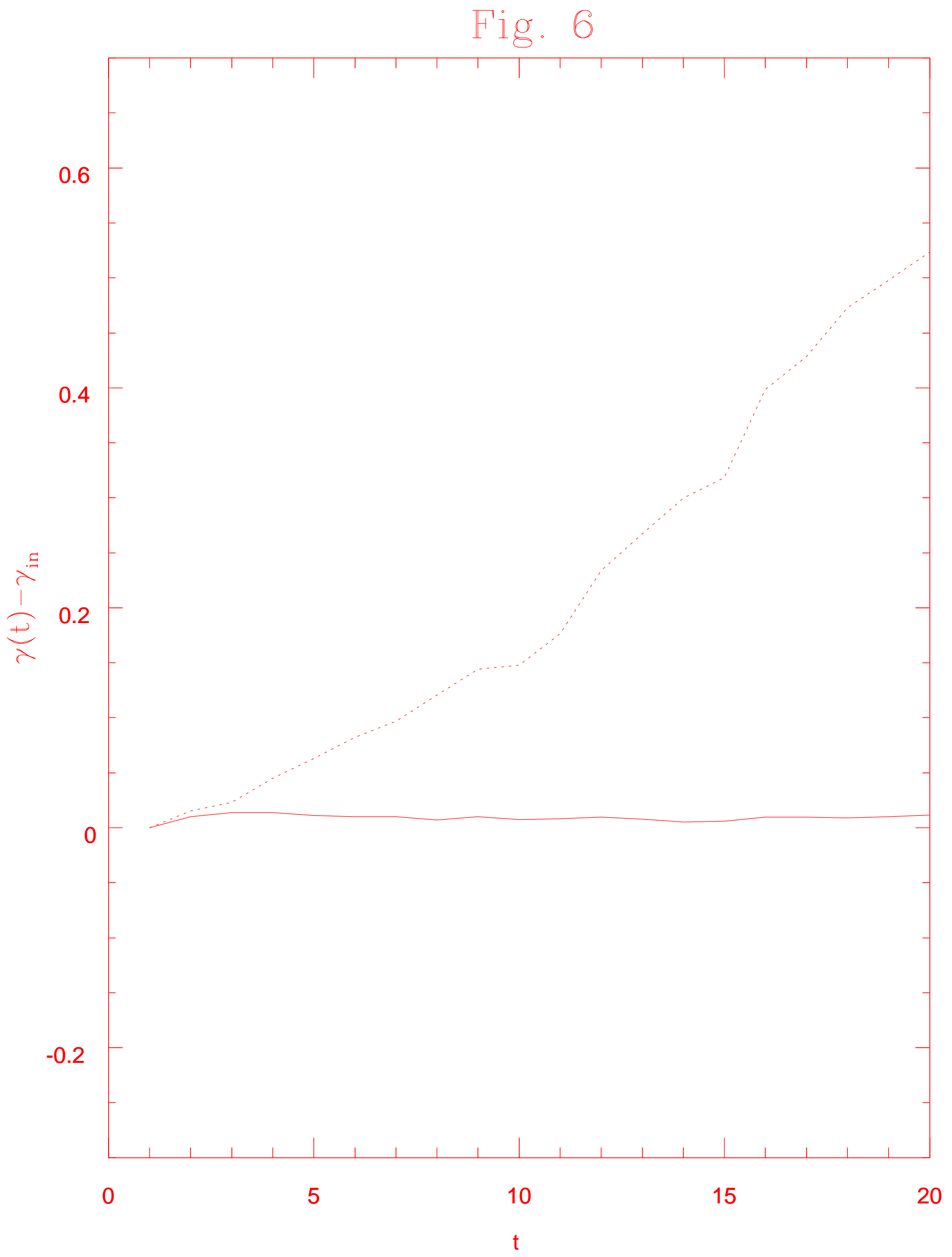




\begin{tabular}{|c|c|c|c|c|}
\hline & $z_{i n}$ & $V_{\text {in }}(\mathrm{km} / \mathrm{s})$ & $C$ & $f(z)$ \\
\hline Homogeneous Field & $\begin{array}{c}3.2 \text { for } \lambda=0 \text { and } \Omega=1 \\
4.2 \text { for } \lambda_{o}=0.8 \text { and } \Omega_{o}=0.2\end{array}$ & $300 \times\left(1+z_{\text {in }}\right)$ & 1 & {$\left[\frac{1+z}{1+z_{\text {in }}}\right]^{4}$} \\
\hline Virialized Structures & 2.2 & 480 & $270\left(t_{\text {in }} / t_{o}\right) \approx 45$ & {$\left[\frac{1+z}{1+z_{\text {in }}}\right]^{(2 n+1) /(n+3)}$} \\
\hline Large Scale Structures & 2.3 & 300 & $5\left(1+z_{i n}\right)^{(D-3) / 2}$ & {$\left[\frac{1+z}{1+z_{\text {in }}}\right]^{(3+D) / 2}$} \\
\hline
\end{tabular}

Table 1: The parameters for the aggregation rate given in equation (2.7) and used in the computation are tabulated for the different galaxy environments discussed in the text; $z_{i n}$ is the starting redshift, $V_{\text {in }}$ is the initial galaxy relative velocity, $C$ is the initial effective density contrast in each environment, and $f(z)$ is the evolutionary factor of the aggregation rate. $D$ defines the dimensionality of the LSS where galaxies reside $(D=1$ for filaments, $D=2$ for sheet-like structures). 